\title{
Generalized Bogoliubov transformation for confined fields: Applications for the Casimir effect
}

\author{
J. C. da Silva, ${ }^{1,2}$ F. C. Khanna, ${ }^{3,4}$ A. Matos Neto, ${ }^{1}$ and A. E. Santana ${ }^{1,3}$ \\ ${ }^{1}$ Instituto de Física, Universidade Federal da Bahia, Campus de Ondina, 40210-340, Salvador, Bahia, Brazil \\ ${ }^{2}$ Centro Federal de Educação Tecnológica da Bahia, Rua Emídio Santos, 40000-900, Salvador, Bahia, Brazil \\ ${ }^{3}$ Physics Department, Theoretical Physics Institute, University of Alberta, Edmonton, Alberta, Canada T6G 2J1 \\ ${ }^{4}$ TRIUMF, 4004, Westbrook Mall, Vancouver, British Columbia, Canada V6T 2 A3
}

(Received 1 May 2002; Revised 2 August 2002; published 12 November 2002)

\begin{abstract}
The Bogoliubov transformation in thermofield dynamics, an operator formalism for the finite-temperature quantum field theory, is generalized to describe a field in arbitrary confined regions of space and time. Starting with the scalar field, the approach is extended to the electromagnetic field and the energy-momentum tensor is written via the Bogoliubov transformation. In this context, the Casimir effect is calculated for zero and nonzero temperature, and therefore it can be considered as a vacuum condensation effect of the electromagnetic field. This aspect opens an interesting perspective for using this procedure as an effective scheme for calculations in the studies of confined fields, including interacting fields.
\end{abstract}

DOI: 10.1103/PhysRevA.66.052101

PACS number(s): 12.20.Ds, 11.10.Wx, 11.10.Ef

\section{INTRODUCTION}

A way to treat the effect of temperature in quantum field theory is, for instance, through the Matsubara formalism $[1,2]$, which is based on a formal substitution of time, say $t$, by a complex time, say $i \tau$. In this imaginary time scheme, the temperature emerges as a consequence of a compactification of the field in a finite interval on the time axis, $0<\tau$ $<\beta$, where $\beta$ is the inverse of temperature (we take the Boltzmann constant as $k_{B}=1$; thus $\left.\beta=1 / k_{B} T=1 / T\right)$. This compactification effect of time coordinates has been generalized and associated with space confinement, for instance, of the electromagnetic field through the notion of the image method for a Green function [3,4]. In addition, in the realm of Euclidean theories, a generalization of the Matsubara formalism has been carried out to take into account spatial confinement of the scalar field in the $\lambda \phi^{4}$ approach using the Epstein-Hurwitz zeta functions [5-7].

On the other hand, as an alternative finite-temperature quantum field theory, Takahashi and Umezawa introduced the so-called thermofield dynamics (TFD) approach [8-17], in order to handle finite temperatures with a real time operator formalism $[17,18]$. TFD is based on two elements. The first one is a doubling in the Fock space $\mathcal{H}$ of the original field system, giving rise to an expanded Fock space denoted by $\mathcal{H}_{T}=\mathcal{H} \otimes \widetilde{\mathcal{H}}$. This doubling, in terms of mappings in $\mathcal{H}_{T}$, is defined by what are called the tilde (or dual) conjugation rules, associating each operator in $\mathcal{H}$, say $a$, to two operators in $\mathcal{H}_{T}$, say $A=a \otimes 1$ and $\widetilde{A}=1 \otimes a$, such that the physical variables are described by the nontilde operators. The next basic ingredient of TFD is a Bogoliubov transformation, introducing a rotation in the tilde and nontilde variables, such that the temperature effect emerges from a condensed state.

When TFD is compared with the imaginary time (Matsubara) formalism, the Bogoliubov transformation works by confining the field in a restricted region of the time axis via the notion of a condensate. Here, our main goal is to develop a generalization of TFD to extend the compactification of an arbitrary field for regions in space through the notion of con- densate. This possibility finds support and should be useful in different contexts, in particular when associated with the vacuum properties of the electromagnetic field via the Casimir effect.

The Casimir effect arises from the fluctuation of the vacuum state of fields defined in space-time manifolds with nontrivial topologies [19-27]. Nowadays there is a prominent interest in the Casimir effect, as a consequence of the fact that in 1997 the Casimir force was measured with a precision of a few percent [28-32]. This result points in particular to a practical use of the Casimir force in nano- and microtechnologies [30,32]. Nevertheless, the finitetemperature corrections in the Casimir effect are one aspect which still demands more theoretical development and needs greater experimental accuracy. Indeed, for example, only recently have the implications of temperature been analyzed in the context of the classical limit of the Casimir effect [3335], although, as emphasized by Mehra long ago [36], the temperature effect is significant for plates separated by a distance of the order of micrometers.

Brown and Maclay [3] treated temperature in the Casimir effect from a full finite-temperature quantum field theory point of view. In their approach the energy-momentum tensor for the electromagnetic field is written in the context of the imaginary time formalism and the image method procedure is used to calculate the propagator between two parallel plates. As a result the temperature effect emerges on the time axis by using a set of images for the propagator. In this calculation, all infinite images are summed up. A set of them (also infinite in number and called odd images), associated with an attempt to make the formalism somewhat covariant, gives zero contribution by a resummation of the infinite series but only at the end of the calculation. This remains as a difficulty, elucidated in the present formalism, since the algebraic manipulations are numerous and there is no available criterion to specify which set of images gives the proper contribution in any specific case $[3,4]$. Here we overcome these difficulties by using a generalization of TFD via an analytic continuation of the Bogoliubov transformations. In this case, we are not concerned with images, such that the 
sum over odd images is no longer needed. Furthermore, the calculations are carried out in a natural covariant way. These aspects represent an ease of calculation, following a rigorous procedure, with an unexpected elucidation of the whole process in a confined space; the Casimir force, derived via the Bogoliubov transformation, can be thought of as a vacuum condensation effect of the electromagnetic field.

We have also applied the method to the situation of one plate made of metal and the other of permeable material (the Casimir-Boyer model) [37]. The interest for this system lies in the fact that we have a repulsive Casimir force, and this type of force has not been satisfactorily studied, although it is a fundamental ingredient for using the Casimir effect associated with technological nanodevices [38]. Furthermore, the Casimir-Boyer model has been only recently addressed in more detail in the literature, in particular in the study of temperature effects $[37,39,40]$. Regarding this system, the results derived here include an explicit expression for the energy-momentum tensor and an analysis of thermodynamics functions such as the Helmholtz free energy and entropy. The results thus obtained open the interesting perspective of using this procedure as an effective scheme for calculations in the studies of different confined fields, including nonAbelian gauge fields like QCD. In other words, this generalized TFD formalism identifies the Casimir effect as giving a direct and clear picture of the vacuum as it displays its properties for differents fields including the non-Abelian field.

In order to proceed, in Sec. II the notation describing the basic elements of TFD is set forth, and the generalized Bogoliubov transformation is introduced for the scalar and the electromagnetic field. The subsequent sections are dedicated to applications. In Sec. III, a thermal stress-energy tensor for the electromagnetic field is derived, and the results are compared with those from the imaginary time formalism. In Sec. IV, the Casimir effect at zero and nonzero temperature is derived. In this case we consider the field constrained between two parallel plates (both made of either conducting or permeable material). The mixed situation, the Casimir-Boyer model $[37,39,40]$, in which there is one conducting and one permeable plate, is discussed in Sec. V. Our final remarks and conclusions are presented in Sec. VI.

\section{TFD AND GENERALIZED BOGOLIUBOV TRANSFORMATION}

Thermofield dynamics is introduced by assuming that the set of operators in a field theory can be given in the form $\mathcal{L}_{T}=\{A, B, C, \ldots, \widetilde{A}, \widetilde{B}, \widetilde{C}, \ldots\}$, defined in the Hilbert space $\mathcal{H}_{T}=\mathcal{H} \otimes \widetilde{\mathcal{H}}$ with elements $|\Phi\rangle=|\phi, \widetilde{\phi}\rangle$. The action of generic operators $A$ and $\widetilde{A}$ on $|\Phi\rangle$ is specified by

$$
\begin{aligned}
& A|\Phi\rangle \equiv a \otimes 1(|\phi\rangle \otimes\langle\phi|)=(a|\phi\rangle) \otimes\langle\phi|, \\
& \widetilde{A}|\Phi\rangle=1 \otimes a(|\phi\rangle \otimes\langle\phi|)=|\phi\rangle \otimes\langle\phi| a^{\dagger},
\end{aligned}
$$

where the operator $a$ is defined in the usual Hilbert space $\mathcal{H}$ with $|\phi\rangle \in \mathcal{H}$ (we follow the usual notation, which is introduced via the identification $A=a$ and $\widetilde{A}=\widetilde{a}$ ). The tilde (or dual) conjugation rules are then a mapping $\sim \mathcal{~}: A \rightarrow \widetilde{A}$, specified by the relations (which can be derived from general algebraic properties $[41,42]$ )

$$
\begin{aligned}
\left(A_{i} A_{j}\right) & \sim \widetilde{A}_{i} \widetilde{A}_{j}, \\
\left(c A_{i}+A_{j}\right) & \sim c^{*} \widetilde{A}_{i}+\widetilde{A}_{j}, \\
\left(A_{i}^{\dagger}\right) & =\left(\widetilde{A}_{i}\right)^{\dagger}, \\
\left(\widetilde{A}_{i}\right) & \sim A_{i}, \\
{\left[\widetilde{A}_{i}, A_{j}\right] } & =0 .
\end{aligned}
$$

The physical variables are described by the nontilde operators and thermal variables are introduced by a Bogoliubov transformation defined by the following procedure. For an arbitrary bosonic operator $A$ we define

$$
\left(A^{a}\right)=\left(\begin{array}{c}
A \\
-\widetilde{A}^{\dagger}
\end{array}\right), \quad\left(\quad A^{a \dagger}\right)=\left(A^{\dagger}, \widetilde{A}\right) .
$$

The Bogoliubov transformation is defined as a $2 \times 2$ matrix,

$$
\mathcal{B}=\left(\begin{array}{cc}
u(\alpha) & -v(\alpha) \\
-v(\alpha) & u(\alpha)
\end{array}\right),
$$

where $u^{2}(\alpha)-v^{2}(\alpha)=1$, with $\alpha$ being a parameter specifying the rotation between tilde and nontilde variables. For instance, for the case of the creation $\left(a^{\dagger}, \tilde{a}^{\dagger}\right)$ and destruction $(a, \tilde{a})$ boson operators, we have the extended (doubled) algebra

$$
\begin{aligned}
& {\left[a, a^{\dagger}\right]=\left[\tilde{a}, \tilde{a}^{\dagger}\right]=1,} \\
& {\left[a^{\dagger}, \tilde{a}\right]=\left[a^{\dagger}, \tilde{a}^{\dagger}\right]=[a, \tilde{a}]=\left[a, \tilde{a}^{\dagger}\right]=0 .}
\end{aligned}
$$

Then the algebraic rules for the thermal bosonic operators are written as $\left[a^{a}(\alpha), a^{b \dagger}(\alpha)\right]=\delta^{a b}, a, b=1,2$, such that $a^{a}=\left(\mathcal{B}^{-1}\right)^{a b} a^{b}(\alpha)$ and $a^{a \dagger}=a^{b}(\alpha) \mathcal{B}^{b a}$. Writing explicitly, we have

$$
\begin{gathered}
a=u(\alpha) a(\alpha)+v(\alpha) \tilde{a}^{\dagger}(\alpha), \\
\tilde{a}=u(\alpha) \tilde{a}(\alpha)+v(\alpha) a^{\dagger}(\alpha), \\
a^{\dagger}=u(\alpha) a^{\dagger}(\alpha)+v(\alpha) \tilde{a}(\alpha), \\
\tilde{a}^{\dagger}=u(\alpha) \tilde{a}^{\dagger}(\alpha)+v(\alpha) a(\alpha),
\end{gathered}
$$

The thermal average is given by taking the vacuum average $|0, \widetilde{0}\rangle$ of the thermal nontilde variables. For instance, for the particular case of the bosonic number operator $n=a^{\dagger} a$, the thermal distribution is given by 


$$
\left.n(\beta)=\langle 0, \widetilde{0}\rangle\left|a^{\dagger}(\alpha) a(\alpha)\right| 0, \widetilde{0}\right\rangle \equiv \frac{1}{e^{\beta \epsilon}-1}=\sum_{n=1}^{\infty} e^{-\beta \varepsilon n}
$$

This result is readily derived if the parameter $\alpha$ is taken to be the temperature, $\alpha=\beta$, such that

$$
\begin{aligned}
& u(\beta)=\frac{1}{\left[1-e^{-\beta \epsilon}\right]^{1 / 2}}, \\
& v(\beta)=\frac{1}{\left[e^{\beta \epsilon}-1\right]^{1 / 2}} .
\end{aligned}
$$

However, observe that $\alpha=\beta$ is a particular choice, and other possibilities for $\alpha$ can be considered as will be seen in the next sections.

The same scheme is generalized for a quantum field. In the case of the free scalar field, since we have equations of motion for the tilde and nontilde variables, the $\alpha$-dependent Klein-Gordon field theory is given by the Lagrangian

$$
\begin{aligned}
\hat{L}= & \frac{1}{2} \partial_{\mu} \phi(x ; \alpha) \partial^{\mu} \phi(x ; \alpha)-\frac{m^{2}}{2} \phi(x ; \alpha)^{2} \\
& -\frac{1}{2} \partial_{\mu} \widetilde{\phi}(x ; \alpha) \partial^{\mu} \widetilde{\phi}(x ; \alpha)+\frac{m^{2}}{2} \widetilde{\phi}(x ; \alpha)^{2},
\end{aligned}
$$

where the metric $g^{\mu \lambda}$ is $\operatorname{such}$ that $\operatorname{diag}\left(g^{\mu \lambda}\right)=(1,-1,-1$, $-1)$. This Lagrangian gives rise to the equations of motion

$$
\left(\partial_{\mu} \partial^{\mu}+m^{2}\right) \phi(x ; \alpha)=0 \text { and }\left(\partial_{\mu} \partial^{\mu}+m^{2}\right) \widetilde{\phi}(x ; \alpha)=0 .
$$

Therefore, in TFD the Lagrangian can be written as $\hat{L}=L$ $-\widetilde{L}$ and in consequence the Hamiltonian is $\hat{H}=H-\widetilde{H}$ (this is a general result that can be used for every field). The two-point Green function for the $\alpha$ scalar field is defined, then, by

$$
\begin{aligned}
G\left(x-x^{\prime} ; \alpha\right)^{(a b)} & =\langle 0, \widetilde{0}| T\left[\phi(x ; \alpha)^{a} \phi\left(x^{\prime} ; \alpha\right)^{b}|0, \widetilde{0}\rangle\right. \\
& =\frac{1}{(2 \pi)^{4}} \int d^{4} k G(k ; \alpha)^{a b} e^{i k\left(x-x^{\prime}\right)},
\end{aligned}
$$

where

$$
G(k ; \alpha)^{(a b)}=\mathcal{B}^{-1}\left(k_{0} ; \alpha\right) G_{0}(k)^{a b} \mathcal{B}\left(k_{0} ; \alpha\right),
$$

with

$$
\mathcal{B}(k, \alpha)=\left(\begin{array}{cc}
u(k, \alpha) & -v(k, \alpha) \\
-v(k, \alpha) & u(k, \alpha)
\end{array}\right)
$$

and

$$
\begin{aligned}
\left(G_{0}(k)^{a b}\right) & =\left(\begin{array}{cc}
G_{0}(k) & 0 \\
0 & \widetilde{G}_{0}(k)
\end{array}\right) \\
& =\left(\begin{array}{cc}
\frac{1}{k^{2}-m^{2}+i \epsilon} & 0 \\
0 & \frac{-1}{k^{2}-m^{2}-i \epsilon}
\end{array}\right) .
\end{aligned}
$$

Then we have

$$
\begin{aligned}
G(k ; \alpha)^{(a b)} & =\mathcal{B}(k, \alpha) G(k)^{(a b)} \mathcal{B}(k, \alpha) \\
& =\left(\begin{array}{ll}
G(k ; \alpha)^{11} & G(k ; \alpha)^{12} \\
G(k ; \alpha)^{21} & G(k ; \alpha)^{22}
\end{array}\right),
\end{aligned}
$$

with

$$
\begin{aligned}
G(k ; \alpha)^{11} & =G_{0}(k)+v^{2}(k, \alpha)\left[G_{0}(k)+\widetilde{G}_{0}(k)\right], \\
G(k ; \alpha)^{12} & =G(k ; \alpha)^{21} \\
& =v(k, \alpha)\left[1+v^{2}(k, \alpha)\right]^{1 / 2}\left[G_{0}(k)+\widetilde{G}_{0}(k)\right], \\
G(k ; \alpha)^{22} & =\widetilde{G}_{0}(k)+v^{2}(k, \alpha)\left[G_{0}(k)+\widetilde{G}_{0}(k)\right] .
\end{aligned}
$$

The physical information is given by $G(k ; \alpha)^{11}$.

Using the definition of $\mathcal{B}\left(k_{0}, \alpha\right)$ given in Eq. (20), with $n(k, \alpha=\beta)=v(k, \alpha=\beta)^{2}=1 /\left[e^{\beta k_{0}}-1\right], \quad$ and $u(k, \alpha=\beta)^{2}$ $=v(k, \alpha=\beta)^{2}+1=1 /\left[1-e^{-\beta k_{0}}\right]$, the components of $G(k ; \beta)^{a b}$ read

$$
\begin{aligned}
G(k ; \beta)^{(11)} & =\frac{1}{k^{2}-m^{2}+i \epsilon}-2 \pi i n\left(k_{0} ; \beta\right) \delta\left(k^{2}-m^{2}\right), \\
G(k ; \beta)^{(22)} & =\frac{-1}{k^{2}-m^{2}-i \epsilon}-2 \pi i n\left(k_{0} ; \beta\right) \delta\left(k^{2}-m^{2}\right), \\
G(k ; \beta)^{(12)} & =G(k ; \beta)^{21} \\
& =-2 \pi i\left[n\left(k_{0} ; \beta\right)+n\left(k_{0} ; \beta\right)^{2}\right]^{1 / 2} \delta\left(k^{2}-m^{2}\right),
\end{aligned}
$$

where $n\left(k_{0} ; \beta\right)=u(k, \alpha=\beta)^{2}$ is the boson distribution function. Notice that the physical propagator $G(k ; \beta)^{11}$ is a well known result derived by the Matsubara method.

The components of

$$
G\left(x-x^{\prime}\right)^{(a b)}=\langle 0, \widetilde{0}| T\left[\phi(x)^{a} \phi\left(x^{\prime}\right)^{b}|0, \widetilde{0}\rangle\right.
$$

for a massless bosonic field can be explicitly written, by using Eq. (21), as

$$
G_{0}^{(11)}\left(x-x^{\prime}\right) \equiv G_{0}\left(x-x^{\prime}\right)=-\frac{i}{(2 \pi)^{2}} \frac{1}{\left(x-x^{\prime}\right)^{2}+i \eta}
$$




$$
G_{0}^{(22)}\left(x-x^{\prime}\right) \equiv \widetilde{G}_{0}\left(x-x^{\prime}\right)=\frac{i}{(2 \pi)^{2}} \frac{1}{\left(x-x^{\prime}\right)^{2}-i \eta}
$$

and $G_{0}^{(12)}\left(x-x^{\prime}\right)=G_{0}^{(21)}\left(x-x^{\prime}\right)=0$. These results will be useful in the following development.

Now we consider the case of an electromagnetic field. Following the tilde conjugation rules, the doubled operator describing the energy-momentum tensor of the electromagnetic field is, then, given by

$$
T^{\mu \lambda(a b)}=-F^{\mu \alpha(a b)} F_{\alpha}^{\lambda(a b)}+\frac{1}{4} g^{\mu \lambda} F_{\beta \alpha}^{(a b)} F^{\alpha \beta(a b)},
$$

where the nontensorial indices $a, b=1,2$ are defined according to the doubled notation given in Eqs. (8),

$$
F_{\mu \nu}^{(a b)}=\partial_{\mu} A_{\nu}^{a}-\partial_{\nu} A_{\mu}^{b} .
$$

The doubled free-photon propagator is thus given by

$$
\begin{aligned}
i D_{\alpha \beta}^{(a b)}\left(x-x^{\prime}\right) & =\left\langle 0, \widetilde{0}\left|T\left[A_{\alpha}^{a}(x) A_{\beta}^{b}(x)\right]\right| 0, \widetilde{0}\right\rangle \\
& =g_{\alpha \beta} G_{0}^{(a b)}\left(x-x^{\prime}\right),
\end{aligned}
$$

where the nonzero components of $G_{0}^{(a b)}\left(x-x^{\prime}\right)$ are given in Eqs. (22) and (23).

The vacuum average of the energy-momentum tensor reads

$$
\begin{aligned}
\left\langle 0, \widetilde{0}\left|T^{\mu \nu(a b)}\right| 0, \widetilde{0}\right\rangle= & -i\left\{\Gamma^{\mu \nu}\left(x, x^{\prime}\right) G_{0}^{(a b)}\left(x-x^{\prime}\right)\right. \\
& \left.+2\left(\eta^{\mu} \eta^{\nu}-\frac{1}{4} g^{\mu \nu}\right) \delta\left(x-x^{\prime}\right) \delta^{a b}\right\}\left.\right|_{x \rightarrow x^{\prime}},
\end{aligned}
$$

where $\eta^{\mu}=(1,0,0,0)$ and

$$
\Gamma^{\mu \nu}\left(x, x^{\prime}\right)=2\left(\partial^{\mu} \partial^{\prime} \underline{\nu} \frac{1}{4} g^{\mu \nu} \partial^{\rho} \partial_{\rho}^{\prime}\right)
$$

Inspired by the usual Casimir prescription, at this point we introduce the tensor $T^{\mu \lambda(a b)}(\alpha)$ by

$$
\begin{aligned}
T_{\text {Cas }}^{\mu \lambda(a b)}(x ; \alpha)= & \left\langle 0, \widetilde{0}\left|T^{\mu \lambda(a b)}(x ; \alpha)\right| 0, \widetilde{0}\right\rangle \\
& -\left\langle 0, \widetilde{0}\left|T^{\mu \lambda(a b)}(x)\right| 0, \widetilde{0}\right\rangle
\end{aligned}
$$

where

$$
\begin{aligned}
\left\langle 0, \widetilde{0}\left|T^{\mu \lambda(a b)}(x ; \alpha)\right| 0, \widetilde{0}\right\rangle= & -i\left\{\Gamma^{\mu \lambda}\left(x, x^{\prime}\right) G^{(a b)}\left(x-x^{\prime} ; \alpha\right)\right. \\
& +2\left(\eta^{\mu} \eta^{\lambda}-\frac{1}{4} g^{\mu \lambda}\right) \\
& \left.\times \delta\left(x-x^{\prime}\right) \delta^{a b}\right\}\left.\right|_{x \rightarrow x^{\prime}} .
\end{aligned}
$$

As a consequence

$$
T_{\text {Cas }}^{\mu \lambda(a b)}(x ; \alpha)=-\left.i\left\{\Gamma^{\mu \lambda}\left(x, x^{\prime}\right) \bar{G}^{(a b)}\left(x-x^{\prime} ; \alpha\right)\right\}\right|_{x \rightarrow x^{\prime}},
$$

where

$$
\bar{G}^{(a b)}\left(x-x^{\prime} ; \alpha\right)=\frac{1}{(2 \pi)^{4}} \int d^{4} k e^{i k\left(x-x^{\prime}\right)} \bar{G}^{(a b)}(k ; \alpha),
$$

such that

$$
\begin{aligned}
\bar{G}^{(11)}(k ; \alpha) & =\bar{G}^{(22)}(k ; \alpha)=v^{2}(k, \alpha)\left[G_{0}(k)+\widetilde{G}_{0}(k)\right], \\
\bar{G}^{(12)}(k ; \alpha) & =\bar{G}^{(21)}(k ; \alpha) \\
& =v(k, \alpha)\left[1+v^{2}(k, \alpha)\right]^{1 / 2}\left[G_{0}(k)+\widetilde{G}_{0}(k)\right] .
\end{aligned}
$$

Let us write a general form for the Bogoliubov transformation by assuming the following analytical continuation for $v^{2}(k, \alpha)$, given originally in Eq. (16):

$$
v^{2}(k, \alpha) \equiv \sum_{l=1}^{\infty} e^{-i \alpha_{l} \cdot k}
$$

where the notation is

$$
\begin{aligned}
\sum_{l=1}^{\infty} e^{-i \alpha_{l} \cdot k}= & \sum_{l_{0}, l_{1}, l_{2}, l_{3}=1}^{\infty} \exp \left[-i\left(\alpha_{0} l_{0} k_{0}+\alpha_{1} l_{1} k_{1}+\alpha_{2} l_{2} k_{2}\right.\right. \\
& \left.\left.+\alpha_{3} l_{3} k_{3}\right)\right],
\end{aligned}
$$

with $\alpha=\left(\alpha_{0}, \alpha_{1}, \alpha_{2}, \alpha_{3}\right)$ representing a set of parameters to be specified. In the following we use this definition for calculating the physical components of the energy-momentum tensor $T^{\mu \lambda(11)}(x ; \alpha)$ in different situations.

\section{TFD STRESS-ENERGY TENSOR AND TEMPERATURE}

As a basic result let us first calculate the temperature effect for the electromagnetic field using this TFD approach. For this proposal we assume that $\alpha_{0}=i \beta=i / T$ and $\alpha_{1}=\alpha_{2}$ $=\alpha_{3}=0$ in Eq. (28). In this case we have

$$
\begin{aligned}
\bar{G}^{(11)}\left(x-x^{\prime} ; \alpha\right)= & \frac{1}{(2 \pi)^{4}} \int d^{4} k e^{i k\left(x-x^{\prime}\right)} \bar{G}^{(11)}(k ; \alpha) \\
= & \frac{1}{(2 \pi)^{4}} \int d^{4} k e^{i k\left(x-x^{\prime}\right)} \sum_{j=1}^{\infty} e^{\beta j k_{0}\left[G_{0}(k)\right.} \\
& \left.+\widetilde{G}_{0}(k)\right]=2 \sum_{j=1}^{\infty} G_{0}\left(x-x^{\prime}-i \beta j n\right),
\end{aligned}
$$

where $n^{\mu}=(1,0,0,0)$. Using this result in Eq. (27) we find 


$$
\begin{aligned}
T^{\mu \lambda(11)}(x ; \alpha)= & -\left.i\left\{\Gamma^{\mu \lambda}\left(x, x^{\prime}\right) \bar{G}^{(11)}\left(x-x^{\prime} ; \alpha\right)\right\}\right|_{x \rightarrow x^{\prime}} \\
= & -i\left\{4\left(\partial^{\mu} \partial^{\prime \lambda}-\frac{1}{4} g^{\mu \nu} \partial^{\rho} \partial_{\rho}^{\prime}\right)\right. \\
& \left.\times \sum_{j=1}^{\infty} G_{0}\left(x-x^{\prime}-i \beta j n\right)\right\}\left.\right|_{x \rightarrow x^{\prime}} \\
= & -\frac{2}{\pi^{2}} \sum_{j=1}^{\infty} \frac{g^{\mu \lambda}-n^{\mu} n^{\lambda}}{(j \beta)^{4}} \\
= & -\frac{1}{45} \pi^{2} T^{4}\left(g^{\mu \lambda}-n^{\mu} n^{\lambda}\right) .
\end{aligned}
$$

Therefore, the energy density of the photon gas is the well known result $E(T)=T^{00(11)}(x ; \alpha)$, which leads to the relation for blackbody radiation, i.e.,

$$
E(T)=\frac{1}{15} \pi^{2} T^{4}
$$

We find that the condensation effect introduced via the Bogoliubov transformation is equivalent, in the imaginary time formalism, to images displaced in time by $i j \beta$, giving rise to a cutoff in the time axis $[3,4]$. In the next section we will see that this condensation produces a cutoff on the space axes.

\section{COMPACTIFICATION IN SPACE-TIME AND THE CASIMIR EFFECT}

In this section we use the formalism developed in Sec. II to derive the Casimir effect at zero and nonzero temperature. We proceed with the same prescription for the energymomentum tensor but with a proper definition of the parameter $\alpha$.

\section{A. Casimir effect at zero temperature}

In the last section we derived the temperature effect for the electromagnetic field with a proper choice for the parameters $\alpha_{l}$, which leads to a Green function $\bar{G}^{(11)}\left(x-x^{\prime} ; \alpha_{l}\right)$ written in terms of a modified free Green function $G_{0}(x$ $\left.-x^{\prime}-i \beta j n\right)$. Let us now assume that $\alpha_{0}=\alpha_{1}=\alpha_{2}=0$ and $\alpha_{3}$, is a real parameter. In this case we have

$$
\begin{aligned}
\bar{G}^{(11)}\left(x-x^{\prime} ; \alpha\right)= & \frac{1}{(2 \pi)^{4}} \int d^{4} k e^{i k\left(x-x^{\prime}\right)} \bar{G}^{(11)}(k ; \alpha) \\
= & \frac{1}{(2 \pi)^{4}} \int d^{4} k e^{i k\left(x-x^{\prime}\right)} \sum_{l=1}^{\infty} e^{i l k_{3} \alpha_{3}\left[G_{0}(k)\right.} \\
& \left.+\widetilde{G}_{0}(k)\right]=2 \sum_{l=1}^{\infty} G_{0}\left(x-x^{\prime}-\alpha_{3} l z\right)
\end{aligned}
$$

Observe from this expression that if we define $\alpha_{3}=2 d$ the sum over $l$ defines the nontrivial part of the Green function that leads to the Dirichlet boundary condition for the electromagnetic field, considering two conducting parallel plates, one at $x_{3}=0$ and the other at $x_{3}=d$ (see Refs. [3,4]). Here the sum is equivalently interpreted as being over half the even images of a photon propagating between two parallel plates (the factor 2 in the sum takes into account the other half of the even images when $x \rightarrow x^{\prime}$ ). This result was first derived by Brown and Maclay, who showed that the contributions due to odd images add to zero $[3,4]$. In our approach this fact is obtained explicitly without reference to images, and the cancellation of the equivalent odd images is a result of the tilde-propagator contribution. Then the nature of the boundary conditions over the electromagnetic field and the associated Green function are the prescription to define the physical content of the parameters $\alpha$.

Using Eq. (31) in Eq. (27), with $\alpha_{3}=2 d$, we find for the energy-momentum tensor

$$
\begin{aligned}
T^{\mu \nu(11)}(x ; d) & =-\left.i\left\{\Gamma^{\mu \nu}\left(x, x^{\prime}\right) \bar{G}^{(11)}\left(x-x^{\prime} ; \alpha\right)\right\}\right|_{x \rightarrow x^{\prime}}=-\left.i\left\{2\left(\partial^{\mu} \partial^{\prime \nu}-\frac{1}{4} g^{\mu \nu} \partial^{\rho} \partial_{\rho}^{\prime}\right) 2 \sum_{l=1}^{\infty} G_{0}\left(x-x^{\prime}-2 d l z\right)\right\}\right|_{x \rightarrow x^{\prime}} \\
& =-\frac{2}{\pi^{2}} \sum_{l=1}^{\infty} \frac{g^{\mu \nu}+4 z^{\mu} z^{\nu}}{(2 l d)^{4}}=-\frac{1}{2^{3} \pi^{2} d^{4}}\left(g^{\mu \nu}+4 z^{\mu} z^{\nu}\right) \sum_{l=1}^{\infty} \frac{1}{l^{4}}=-\frac{\pi^{2}}{180 d^{4}}\left(\frac{1}{4} g^{\mu \nu}+z^{\mu} z^{\nu}\right) .
\end{aligned}
$$

In particular, the component of the Casimir energy $T^{00(11)}(x ; \alpha)=E(d)$ is then given as

$$
E(d)=-\frac{\pi^{2}}{720 d^{4}}
$$

\section{B. Casimir effect at nonzero temperature}

Consider the case $\alpha_{0}=i \beta, \alpha_{1}=\alpha_{2}=0, \quad \alpha_{3}=2 d, n^{\mu}$ $=(1,0,0,0)$, and $z^{\mu}=\left(0,0,0, n_{3}\right)$. In this case we have 


$$
\begin{aligned}
\bar{G}^{(11)}\left(x-x^{\prime} ; \alpha\right)= & \frac{1}{(2 \pi)^{4}} \int d^{4} k e^{i k\left(x-x^{\prime}\right)} \bar{G}^{(11)}(k ; \alpha) \\
= & \frac{1}{(2 \pi)^{4}} \int d^{4} k e^{i k\left(x-x^{\prime}\right)} \sum_{j, l=1}^{\infty} e^{\beta j k_{0}+i l k_{3}(2 d)} \\
& \times\left[G_{0}(k)+\widetilde{G}_{0}(k)\right] \\
= & 2 \sum_{j, l=1}^{\infty} G_{0}\left(x-x^{\prime}-i \beta j n-2 d l z\right) .
\end{aligned}
$$

Using this result in Eq. (27) we find that the energymomentum tensor is

$$
\begin{aligned}
T^{\mu \nu(11)}(x ; d, \beta)= & -\left.i\left\{\Gamma^{\mu \nu}\left(x, x^{\prime}\right) \bar{G}^{(11)}\left(x-x^{\prime} ; \alpha\right)\right\}\right|_{x \rightarrow x^{\prime}} \\
= & -i\left\{2\left(\partial^{\mu} \partial^{\prime \nu}-\frac{1}{4} g^{\mu \nu} \partial^{\rho} \partial_{\rho}^{\prime}\right) 2\right. \\
& \left.\times \sum_{j, l=1}^{\infty} G_{0}\left(x-x^{\prime}-i \beta j n-2 d l z\right)\right\}\left.\right|_{x \rightarrow x^{\prime}} .
\end{aligned}
$$

Notice that if we take in the sum given in Eq. (34) $l=0$ or $j=0$, we recover the terms of the blackbody radiation, Eq. (30), and the Casimir effect at zero temperature, Eq. (33). Thus in this case the energy-momentum tensor is

$$
\begin{aligned}
T^{\mu \nu(11)}(x ; d, \beta)= & -\frac{4}{\pi^{2}} \sum_{j, l=0^{\prime}}^{\infty}\left\{\frac{g^{\mu \nu}}{\left[(2 l d)^{2}+(j \beta)^{2}\right]^{2}}\right. \\
& \left.+\frac{4(2 l d)^{2} z^{\mu} z^{\nu}-(j \beta)^{2} n^{\mu} n^{\nu}}{\left[(2 l d)^{2}+(j \beta)^{2}\right]^{2}}\right\},
\end{aligned}
$$

where the notation $j, l=0^{\prime}$ is used to emphasize that the term for $l=j=0$ is not included in the sum [actually this is a divergent term that was subtracted in Eq. (26)].

Following Brown and Maclay [3], we define $\xi=d / \beta$,

$$
f(\xi)=-\frac{1}{4 \pi^{2}} \sum_{j, l=0^{\prime}}^{\infty} \frac{(2 \xi)^{4}}{\left[(2 l \xi)^{2}+(j)^{2}\right]^{2}},
$$

and

$$
s(\xi)=-\frac{d}{d \xi} f(\xi)=\frac{2^{4}}{\pi^{2}} \sum_{j, l=0^{\prime}}^{\infty} \frac{\xi^{3} j^{2}}{\left[(2 l \xi)^{2}+(j)^{2}\right]^{3}},
$$

resulting in

$$
\begin{aligned}
T^{\mu \nu(11)}(x ; d, \beta)= & \frac{1}{d^{4}} f(\xi)\left(g^{\mu \nu}+4 z^{\mu} z^{\nu}\right) \\
& +\frac{1}{\beta d^{3}}\left(n^{\mu} n^{\nu}+z^{\mu} z^{\nu}\right) s(\xi)
\end{aligned}
$$

In particular, the component $T^{00(11)}(x ; d, \beta)=E(d, \beta)$ gives rise to the energy density

$$
E(d, \beta)=\frac{1}{d^{4}}[f(\xi)+\xi s(\xi)] .
$$

Here $f(\xi)$ describes the Helmholtz free-energy density for photons and $s(\xi)$ is the entropy density.

\section{CASIMIR-BOYER MODEL}

In the last section we applied the generalized Bogoliubov transformation to treat the Casimir effect, such that the Green function fulfilled the Dirichlet (Neumann) boundary condition for two conducting (permeable) parallel plates, one at $x_{3}=0$ and the other at $x_{3}=d$. In this section we consider the Casimir-Boyer model [37], corresponding to a mixed situation of plates in which at $x_{3}=0$ we have a conducting plate (Dirichlet boundary conditions) and at $x_{3}=d$ a permeable plate (Neumann boundary conditions). In order to have a Green function satisfying these conditions, we consider $\alpha_{0}$ $=i \beta, \alpha_{1}=\alpha_{2}=0, \quad \alpha_{3}=2 d+\pi / k_{3}, n^{\mu}=(1,0,0,0)$, and $z^{\mu}$ $=(0,0,0,1)$. In this case we have

$$
\begin{aligned}
\bar{G}^{(11)}\left(x-x^{\prime} ; \alpha\right) & =\frac{1}{(2 \pi)^{4}} \int d^{4} k e^{i k\left(x-x^{\prime}\right)} \bar{G}^{(11)}(k ; \alpha)=\frac{1}{(2 \pi)^{4}} \int d^{4} k e^{i k\left(x-x^{\prime}\right)} \sum_{j, l=1}^{\infty}(-1)^{l} e^{\beta j k_{0}+i l k_{3}(2 d)}\left[G_{0}(k)+\widetilde{G}_{0}(k)\right] \\
& =2 \sum_{j, l=1}^{\infty}(-1)^{l} G_{0}\left(x-x^{\prime}-i \beta j n-2 d l z\right) .
\end{aligned}
$$

Using this result in Eq. (27) we find the energy-momentum tensor to be

$$
\begin{aligned}
T^{\mu \nu(11)}(x ; d, \beta) & =-\left.i\left\{\Gamma^{\mu \nu}\left(x, x^{\prime}\right) \bar{G}^{(11)}\left(x-x^{\prime} ; \alpha\right)\right\}\right|_{x \rightarrow x^{\prime}} \\
& =-\left.i\left\{2\left(\partial^{\mu} \partial^{\prime \nu}-\frac{1}{4} g^{\mu \nu} \partial^{\rho} \partial_{\rho}^{\prime}\right) 2 \sum_{j, l=1}^{\infty}(-1)^{l} G_{0}\left(x-x^{\prime}-i \beta j n-2 d l z\right)\right\}\right|_{x \rightarrow x^{\prime}} .
\end{aligned}
$$


As before, if we take the sums starting from $l, j=0$, we include the Casimir effect at zero temperature and the blackbody radiation. Hence, carrying out the calculations in Eq. (36), we find that

$$
\begin{aligned}
T^{\mu \nu(11)}(x ; d, \beta)= & -\frac{4}{\pi^{2}} \sum_{j, l=0^{\prime}}^{\infty}(-1)^{l}\left\{\frac{g^{\mu \nu}}{\left[(2 l d)^{2}+(j \beta)^{2}\right]^{2}}\right. \\
& \left.+\frac{4(2 l d)^{2} z^{\mu} z^{\nu}-(j \beta)^{2} n^{\mu} n^{\nu}}{\left[(2 l d)^{2}+(j \beta)^{2}\right]^{2}}\right\}
\end{aligned}
$$

Observe that for the term $l=0$ the component $T^{00(11)}(x ; d, \beta)$ is the blackbody radiation term given in Eq. (30), and for $j$ $=0$ we have $T^{00(11)}(x ; d, \beta)=E(d)$,

$$
E(d)=\frac{7}{8} \frac{\pi^{2}}{720 d^{4}},
$$

which is the Casimir energy for the Casimir-Boyer model $[37,39,40]$. Notice that this energy corresponds to an attractive force which is $-7 / 8$ of the Casimir energy for plates of the same material.

Using $\xi=d / \beta$, we introduce

$$
\hat{f}(\xi)=-\frac{1}{4 \pi^{2}} \sum_{j, l=0^{\prime}}^{\infty}(-1)^{l} \frac{(2 \xi)^{4}}{\left[(2 l \xi)^{2}+(j)^{2}\right]^{2}},
$$

and

$$
\hat{s}(\xi)=-\frac{d}{d \xi} f(\xi)=\frac{2^{4}}{\pi^{2}} \sum_{j, l=0^{\prime}}^{\infty}(-1)^{l} \frac{\xi^{3} j^{2}}{\left[(2 l \xi)^{2}+(j)^{2}\right]^{3}},
$$

resulting in

$$
\begin{aligned}
T^{\mu \nu(11)}(x ; d, \beta)= & \frac{1}{d^{4}} \hat{f}(\xi)\left(g^{\mu \nu}+4 z^{\mu} z^{\nu}\right) \\
& +\frac{1}{\beta d^{3}}\left(n^{\mu} n^{\nu}+z^{\mu} z^{\nu}\right) s(\xi) .
\end{aligned}
$$

In particular, the component $T^{00(11)}(x ; d, \beta)=E(d, \beta)$ gives rise to the energy density

$$
E(d, \beta)=\frac{1}{d^{4}}[\hat{f}(\xi)+\xi \hat{s}(\xi)] .
$$

Here $\hat{f}(\xi)$ describes the Helmholtz free-energy density for photons and $\hat{s}(\xi)$ is the entropy density.

\section{CONCLUDING REMARKS}

Summarizing, in this work a generalization for the thermofield dynamics formalism is presented, via an analytic continuation of the usual Bogoliubov transformation, in order to describe a field in a confined region in space. We apply the method to calculate the energy-momentum tensor of the electromagnetic field in different situations associated with the Casimir effect, such that in each case some peculiar aspect of the approach is emphasized.
Our aim with the applications was to demonstrate that our calculational method simplifies the study of the Casimir effect considerably, using the notion of covariance throughout the calculations. Furthermore, the case that gives rise to a repulsive force, which is of some interest in the most recent literature [38-40], is dealt within detail, resulting in the following results: (a) an explicit expression for the energymomentum tensor; (b) an explicit calculation of the expressions for the Helmholtz free energy, the internal energy, and the entropy.

In this TFD generalization, the Casimir effect is interpreted as a process of condensation of the electromagnetic field. In the case of the Casimir effect at zero temperature, the quasiparticles are described (for an arbitrary mode) by

$$
\begin{gathered}
a(\alpha)=u(\alpha) a-v(\alpha) \tilde{a}^{\dagger}, \\
\tilde{a}^{\dagger}(\alpha)=u(\alpha) \tilde{a}^{\dagger}-v(\alpha) a,
\end{gathered}
$$

with $a(\alpha)$ and $\tilde{a}$ fulfilling the canonical algebra of the creation and destruction operators, that is, $\left[a(\alpha), a^{\dagger}(\alpha)\right]$ $=\left[\tilde{a}(\alpha), \tilde{a}^{\dagger}(\alpha)\right]=1$. From these operators a vacuum state $|0(\alpha)\rangle$ can be defined, such that $a(\alpha)|0(\alpha)\rangle=0$. Therefore, regarding the operators $a$ and $a^{\dagger}$, the state $|0(\alpha)\rangle$ describes a condensate, as is the case for the temperature in the usual TFD. This provides an unusual insight into the role of the vacuum in developing the Casimir force. Thus not only the notion of the vacuum but also its structure (seen as a condensate) is crucial in producing the Casimir effect. In a broader sense, this notion of condensate is a central aspect throughout the paper, which cannot be derived in the context of the Matsubara formalism.

In Ref. [6], using a modification of the Matsubara approach to treat spontaneous symmetry breaking in compactified $\lambda \phi^{4}$ theory and the superconducting transition temperature in thin films, it is shown how to describe a general space confinement of a field, not necessarily in the ground state as is the case of the Casimir effect. However, our contention is that a more refined understanding of that modified Matsubara method would be interesting, considering theoretical and practical applications. This aspect has been achieved here by using a generalization of TFD, which can be used as well for the systems studied in [6].

The Matsubara formalism has also been applied in the derivation of the so-called Lifshitz formula, describing the Casimir force in real media $\epsilon(\mathbf{x}, \beta)$ (not $\epsilon_{0}=1$ ) [32]. Recently, this formula has been successfully used with the dielectric permittivity, as is given by the Drude model function, to describe the Casimir force at nonzero temperature between real metals [44]. Thus, it would be interesting to analyze the connection of our method and the Matsubara approach more closely. This can be carried out by using functional methods in TFD [43]. In our case, considering the scalar field, we can start with the following definition for the generating functional:

$$
\mathcal{Z}^{a b}=N \exp \left\{\frac{-i}{2} \int(J-J) G\left(x-x^{\prime}, \alpha\right)\left(\begin{array}{c}
J \\
-J
\end{array}\right)\right\} d x d y,
$$


where the matrix $G\left(x-x^{\prime}, \alpha\right)$ is given in Eq. (19). From $\mathcal{Z}^{a b}$ an effective action, say $W$, can then be introduced by $W^{a b}$ $=-i \ln \mathcal{Z}^{a b}$. Taking $a=b=1$, we recover the Matsubara method, and in particular the results given in Eq. (3.10) of Ref. [32], for the effective action under the zeta-functional regularization, the starting point for deriving the aforementioned Lifshitz formula. These aspects regarding the use of the generalized Bogoliubov transformation associated with the Casimir effect for real (not only ideal) media will be discussed in more detail elsewhere.

Ending these remarks, it is worth adding that, as this method based on the Bogoliubov transformation is independent of the type of field involved, it should be useful to analyze the Casimir effect in the case of non-Abelian gauge fields like quantum chromodynamics. In this case the Casimir effect affects the formation of the quark-gluon plasma, and as a consequence the phase transition from a confined to a deconfined state.

\section{ACKNOWLEDGMENTS}

The authors thank Professor M. Revzen for stimulating discussions and for his interest in this work. One of us (A.E.S.) thanks A. P. C. Malbouisson and J. M. C. Malbouisson for interesting discussions. This work was supported by CNPQ of Brazil and NSERC of Canada.
[1] T. Matsubara, Prog. Theor. Phys. 14, 351 (1955).

[2] A. L. Fetter and J. D. Walecka, Quantum Theory of ManyParticles Systems (McGraw-Hill, New York, 1971); A. Das, Finite Temperature Field Theory (World Scientific, Singapore, 1977).

[3] L.S. Brown and G.J. Maclay, Phys. Rev. 184, 1272 (1969).

[4] G. Plunien, B. Müller, and W. Greiner, Phys. Rep. 134, 87 (1986).

[5] A.P.C. Malbouisson and J.M.C. Malbouisson, J. Phys. A 35, 2263 (2001).

[6] A.P.C. Malbouisson, J.M.C. Malbouisson, and A.E. Santana, Nucl. Phys. B 631, 83 (2002); e-print cond-mat/0205176.

[7] A. Elizalde and E. Romeo, J. Math. Phys. 30, 1133 (1989).

[8] Y. Takahashi and H. Umezawa, Collect. Phenom. 2, 55 (1975); [reprinted in Int. J. Mod. Phys. A 10, 1755 (1996)].

[9] H. Umezawa, H. Matsumoto, and M. Tachiki, Thermofield Dynamics and Condensed States (North-Holland, Amsterdam, 1982).

[10] A. Mann, M. Revzen, H. Umezawa, and Y. Yamanaka, Phys. Lett. A 140, 475 (1989).

[11] P.A. Henning, Ch. Becker, A. Lang, and U. Winkler, Phys. Lett. B 217, 211 (1989).

[12] H. Umezawa, Advanced Field Theory: Micro, Macro and Thermal Physics (AIP, New York, 1993).

[13] A.E. Santana and F.C. Khanna, Phys. Lett. A 203, 68 (1995).

[14] A.E. Santana, F.C. Khanna, H. Chu, and C. Chang, Ann. Phys. (N.Y.) 249, 481 (1996).

[15] M.C.B. Andrade, A.E. Santana, and J.D. Vianna, J. Phys. A 33, 4015 (2000).

[16] Proceedings of the IVth International Workshop on Thermal Theory and Its Applications, edited by Y. X. Gui, F. C. Khanna, and Z. B. Su (World Scientific, Singapore, 1996).

[17] R. Kobes, Phys. Rev. D 42, 562 (1990).

[18] M. Le Bellac, Thermal Field Theory (Cambridge University Press, Cambridge, England, 1996).

[19] H.B.G. Casimir, Proc. K. Ned. Akad. Wet. 51, 793 (1948).

[20] P. W. Milonni, The Quantum Vacuum (Academic Press, Boston, 1993).

[21] V. M. Mostepanenko and N. N. Trunov, The Casimir Effect and Its Applications (Clarendon, Oxford, 1997).
[22] K.A. Milton, e-print hep-th/9901011.

[23] F. Caruso, N.P. Neto, B.F. Svaiter, and N.F. Svaiter, Phys. Rev. D 43, 1300 (1991).

[24] B.F. Svaiter and N.F. Svaiter, J. Math. Phys. 32, 175 (1991).

[25] F. Caruso, R. de Paola, and N.F. Svaiter, Int. J. Mod. Phys. A 14, 2077 (1999).

[26] M.V. Cougo-Pinto, C. Farina, and A. Tenório, Braz. J. Phys. 29, 371 (1999).

[27] D.T. Alves, C. Farina, and A.C. Tort, Phys. Rev. A 61, 034102 (2000).

[28] S.K. Lamoreaux, Phys. Rev. Lett. 78, 5 (1997); 81, 5475 (1998).

[29] U. Mohideen and A. Roy, Phys. Rev. Lett. 81, 4549 (1998).

[30] S.K. Lamoreaux, Am. J. Phys. 67, 850 (1999).

[31] The Casimir Effect 50 Years Later, edited by M. Bordag (World Scientific, Singapore, 1999).

[32] M. Bordag, U. Mohideen, and V.M. Mostepanenko, Phys. Rep. 353, 1 (2001).

[33] M. Revzen, R. Opher, M. Opher, and A. Mann, Europhys. Lett. 38, 245 (1997).

[34] M. Revzen, R. Opher, M. Opher, and A. Mann, J. Phys. A 30, 7783 (1997).

[35] J. Feinberg, A. Mann, and M. Revzen, Ann. Phys. (N.Y.) 288, 103 (2001).

[36] J. Mehra, Physica (Amsterdam) 37, 145 (1967).

[37] T.H. Boyer, Phys. Rev. A 9, 2078 (1974).

[38] O. Kenneth, I. Klich, A. Mann, and M. Revzen, Phys. Rev. Lett. 89, 033001 (2002).

[39] J.C. da Silva, A. Matos Neto, H.Q. Plácido, M. Revzen, and A.E. Santana, Physica A 292, 411 (2001).

[40] F.C. Santos, A. Tenório, and A.C. Tort, Phys. Rev. D 60, 105022 (2000).

[41] A.E. Santana, A. Matos Neto, J.D.M. Vianna, and F.C. Khanna, Physica A 280, 405 (2000).

[42] M. de Montigny, F.C. Khanna, and A.E. Santana, Can. Appl. Math. Q. 8, 43 (2000).

[43] G.W. Semenoff and H. Umezawa, Nucl. Phys. B 220, 196 (1983).

[44] G.L. Klimchitskaya and V.M. Mostepanenko, Phys. Rev. A 63, 062108 (2001). 